\title{
Large planar Hall effect in bismuth thin films
}

\author{
Shuo-Ying Yang $\odot$, Kai Chang $\odot,{ }^{*}$ and Stuart S. P. Parkin ${ }^{\dagger}$ \\ Max Planck Institute of Microstructure Physics, Weinberg 2, 06120 Halle (Saale), Germany
}

(Received 23 December 2019; accepted 1 April 2020; published 6 May 2020)

\begin{abstract}
The origin of the planar Hall effect (PHE) in various nonmagnetic semimetals has become a subject of considerable interest, especially in regard to the chiral anomaly that several of these semimetals exhibit. Here, we report a large PHE that exceeds several $\mathrm{m} \Omega \mathrm{cm}$ over a wide range of temperature $T$ and magnetic field $B$ in micron-thick single-crystalline bismuth thin films grown by molecular beam epitaxy techniques. The angular dependence of the PHE and the related anisotropic magnetoresistance (AMR) show complex behaviors as a function of $B$ and $T$. At high temperatures and in modest magnetic fields, the PHE and AMR can be quantitatively explained by a semiclassical transport model based on the well-established elongated electron and hole pockets of the Fermi surface in bismuth. Although these results establish an anisotropic electronic orbital origin of the PHE, we find that when the electric current is oriented along the binary axis of bismuth, the PHE and AMR behaviors can be well described by a model based on the chiral anomaly in Weyl or Dirac semimetals. However, this model cannot account for these behaviors when the current is rather oriented along the bisectrix axis. Thus, the anisotropy of the PHE is a useful test to check on the validity of the chiral anomaly in semimetals.
\end{abstract}

DOI: 10.1103/PhysRevResearch.2.022029

The planar Hall effect (PHE) is the generation of a transverse voltage in a standard Hall bar geometry when an applied magnetic field is rotated in the plane defined by the electric current direction and the Hall electrodes. This effect typically originates from the interaction between the magnetic order and spin-orbit coupling in ferromagnetic metals [1,2], and, indeed, a series of planar Hall sensors for the measurement of weak magnetic fields has been reported [3-6]. In nonmagnetic semimetals, this effect can also be induced by the orbital anisotropy of the electronic structure. For example, bismuth is a semimetal with highly anisotropic pockets in the Fermi surface [7-9], as shown in Fig. 1(a). There are three electron pockets elongated along a direction slightly tilted away from the bisectrix axis and one hole pocket elongated along the trigonal axis. The PHE in bismuth has been briefly reported [10], but detailed quantitative studies are lacking. In addition to the orbital origin, it has recently been proposed that the PHE is a signature of a chiral anomaly in Weyl and Dirac semimetals $[11,12]$. This prediction was quickly followed by a number of experimental demonstrations of PHE in several topological semimetals, including $\mathrm{Cd}_{3} \mathrm{As}_{2}$ [13,14], $\mathrm{MoTe}_{2}$ [15], $\mathrm{ZrTe}_{5}$ [16], $\mathrm{VAl}_{3}$ [17], and GdPtBi [18]. However, a later study suggested that the existence of a chiral anomaly in a semimetal should be tested by referring to parametric

\footnotetext{
*Present address: Beijing Academy of Quantum Information Science 100193, China; changkai@baqis.ac.cn

†stuart.parkin@mpi-halle.mpg.de

Published by the American Physical Society under the terms of the Creative Commons Attribution 4.0 International license. Further distribution of this work must maintain attribution to the author(s) and the published article's title, journal citation, and DOI. Open access publication funded by the Max Planck Society.
}

plots between the anisotropic magnetoresistance (AMR) and the PHE [10]. Several very recent experiments report that the anisotropic orbital magnetoresistance can also significantly contribute to the PHE in topological semimetals, such as $\mathrm{TaP}$ [19], $\mathrm{MoTe}_{2}$ [20], $\mathrm{NiTe}_{2}$ [21], and $\mathrm{PdTe}_{2}$ [22]. Thereby, the approach of distinguishing different origins of PHE in semimetals has become an important issue, which has been appreciated in a recent study [23]. In this Rapid Communication, we report a large PHE that exhibits complex behaviors as a function of temperature and magnetic field in high-quality single-crystalline bismuth thin films. The magnitude of PHE in the bismuth films is comparable to the largest yet reported in topological semimetals. We have explored the origin of such a large PHE in bismuth films by quantitatively comparing PHE and AMR data to two models, one based on the chiral anomaly and one using a semiclassical transport model that reflects the anisotropy of orbital magnetoresistance.

The studies reported here use (111)-oriented bismuth thin films, several microns thick, that were grown using molecular beam epitaxy methods on $\mathrm{BaF}_{2}$ (111) substrates [24]. Both scanning tunneling microscopy (STM) and x-ray diffraction (XRD) measurements confirm the single-crystalline growth with the trigonal axis pointing out of plane [25]. Two groups of Hall bars $(L=180 \mu \mathrm{m} ; \mathrm{W}=60 \mu \mathrm{m} ; d=5.8 \mu \mathrm{m})$ along the binary and bisectrix directions were fabricated using photolithography techniques, as shown in Fig. 1(b). We define $\varphi$ as the angle between the in-plane magnetic field and the binary axis. In this configuration, the in-plane resistivity tensor can be written as

$$
\hat{\rho}(\varphi)=\left(\begin{array}{ll}
\rho_{11}(\varphi) & \rho_{12}(\varphi) \\
\rho_{21}(\varphi) & \rho_{22}(\varphi)
\end{array}\right)
$$

in which $\rho_{11}(\varphi)$ and $\rho_{12}(\varphi)$ are the AMR and PHE in the binary channel, respectively, and $\rho_{22}(\varphi)$ and $\rho_{21}(\varphi)$ are those 
(a)
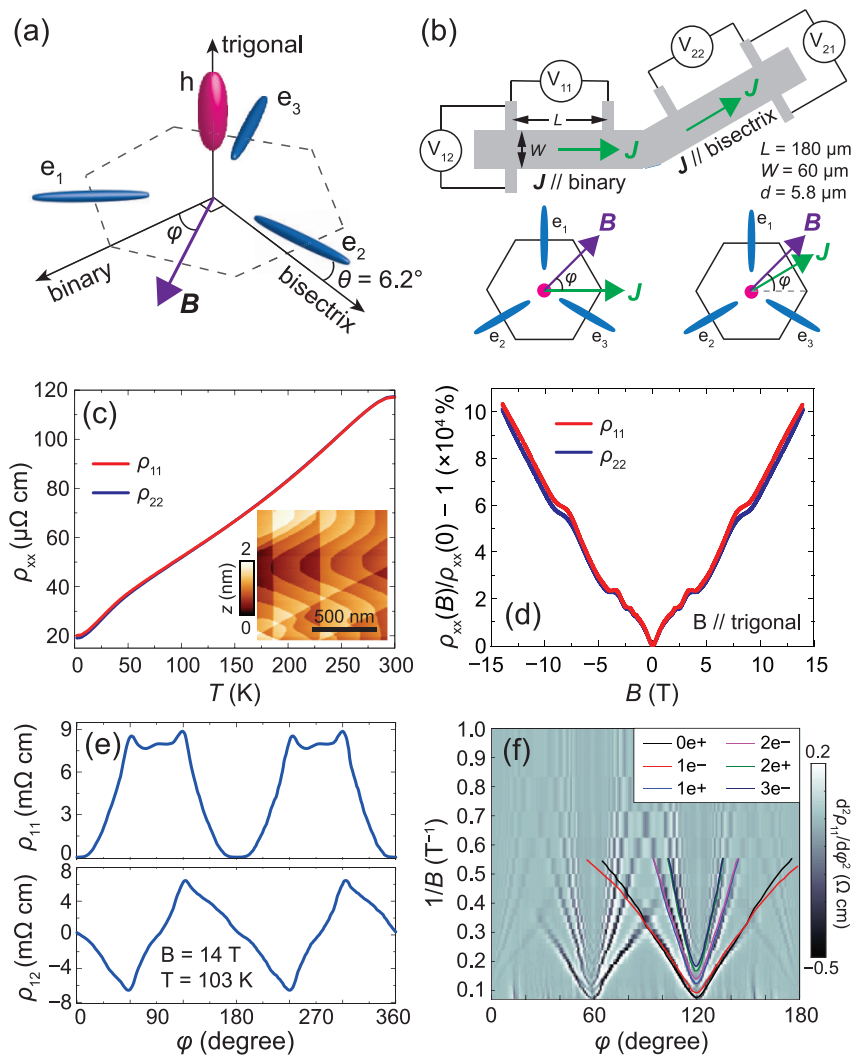

FIG. 1. (a) Schematic diagram of the Fermi pockets of bismuth. The hexagon indicates the projection of the first Brillouin zone on the binary-bisectrix plane. (b) Schematic of the Hall bar devices used in this study. (c) Temperature dependence of the resistivities in the binary $\left(\rho_{11}\right)$ and bisectrix $\left(\rho_{22}\right)$ channels. Inset: STM topography of the as-grown bismuth film. (d) Transverse magnetoresistances with $B$ parallel to the trigonal axis, measured at $2 \mathrm{~K}$. (e) Anisotropic magnetoresistance and planar Hall effect in the binary channel, with the field rotating in the binary-bisectrix plane. (f) Landau level diagram measured in the binary channel at $2 \mathrm{~K}$. The superposed curves indicate the calculated electron Landau levels corresponding to $n=3.85 \times 10^{17} \mathrm{~cm}^{-3}$. The legends " $i \mathrm{e}+/-$ " are the labels of electron Landau levels, in which $i$ is the filling number, and +/indicates the spin state.

in the bisectrix channel. With no magnetic field applied, the temperature dependences of resistivities in both channels exhibit metallic behavior, showing a residual resistance ratio (RRR) of 5.9 [Fig. 1(c)], comparable with the highest-quality bismuth thin films grown by electrodeposition [26]. The transverse magnetoresistance (TMR) with $\boldsymbol{B}$ along the trigonal axis reaches $100000 \%$ at $T=2 \mathrm{~K}$ and $B=14 \mathrm{~T}$ with no sign of saturation [Fig. 1(d)], consistent with the nearly compensating electron and hole carriers. Figure 1(e) shows examples of experimental AMR and PHE curves for the binary channel, measured by rotating the sample in the binary-bisectrix plane at $103 \mathrm{~K}$ and $14 \mathrm{~T}$. Both curves show a period of $\pi$. The maximum in $\rho_{12}$ exceeds $6 \mathrm{~m} \Omega \mathrm{cm}$, a value higher than others reported in a topological semimetal [13-22].

Now we explore the origin of the large PHE. As mentioned above, in a nonmagnetic material the PHE can be a result of the chiral anomaly or an anisotropic orbital magnetoresis- tance. To study if the observed PHE is related to an orbital anisotropy, a semiclassical model that takes the multivalley and anisotropic fermiology of bismuth into account is applied [7]. The model here uses the tensors

$$
\hat{\mu}_{\mathrm{e} 1}=\left(\begin{array}{ccc}
\mu_{1} & 0 & 0 \\
0 & \mu_{2} & \mu_{4} \\
0 & \mu_{4} & \mu_{3}
\end{array}\right), \quad \hat{v}=\left(\begin{array}{ccc}
v_{1} & 0 & 0 \\
0 & v_{1} & 0 \\
0 & 0 & v_{3}
\end{array}\right)
$$

to describe the mobilities of the first electron pocket and the hole pocket. The three bases are along the binary, bisectrix, and trigonal directions, respectively. The mobility tensors of the other electron pockets are obtained from $\hat{\mu}_{\mathrm{e} 2}=\hat{R}_{\varphi}^{-1}(2 \pi / 3) \cdot \hat{\mu}_{\mathrm{e} 1} \cdot \hat{R}_{\varphi}(2 \pi / 3)$ and $\hat{\mu}_{\mathrm{e} 3}=\hat{R}_{\varphi}^{-1}(-2 \pi / 3)$. $\hat{\mu}_{\mathrm{e} 1} \cdot \hat{R}_{\varphi}(-2 \pi / 3)$, in which $\hat{R}_{\varphi}$ is the rotation tensor. At a steady state in a magnetic field, the conductivity tensor can be written as

$$
\hat{\sigma}=\sum_{i=1}^{3} \frac{1}{3} n e\left[\hat{\mu}_{\mathrm{e} i}^{-1}-\hat{B}\right]^{-1}+p e\left[\hat{v}^{-1}+\hat{B}\right]^{-1},
$$

in which $n$ and $p$ are the electron and hole densities, and the magnetic field tensor is given by

$$
\hat{B}=\left(\begin{array}{ccc}
0 & -B_{3} & B_{2} \\
B_{3} & 0 & -B_{1} \\
-B_{2} & B_{1} & 0
\end{array}\right)
$$

The resistivity tensor can be obtained by inverting the conductivity tensor $\hat{\rho}=\hat{\sigma}^{-1}$; the latter contains seven independent parameters: $\mu_{1}, \mu_{2}, \mu_{3}, \nu_{1}, \nu_{3}, n$, and $p$. Note that $\mu_{4}=$ $\left(\mu_{2}-\mu_{3}\right) \tan (2 \theta) / 2$ can be determined by the tilting angle $\theta$ of the electron pockets off the bisectrix axis, which is $6.2^{\circ}$ at $2 \mathrm{~K}$ [8] and $8.3^{\circ}$ at $300 \mathrm{~K}$ [9]. Note that the fielddirection dependence of the element $\rho_{33}$ in the resistivity tensor has been reported previously. Here, we will focus on measurements of $\rho_{11}, \rho_{22}, \rho_{12}$, and $\rho_{21}$ [27].

To minimize the number of fitting parameters, $n$ and $p$ at $2 \mathrm{~K}$ were extracted from the angle-dependent Landau level (LL) diagrams [Fig. 1(f)]. With $\boldsymbol{B}$ rotating in plane, the cross sections of the electron pockets vary rapidly with $\varphi$, while that of the hole pocket changes moderately. Therefore, the dispersive LLs revealed in Fig. 1(f) are from the electron pockets. Comparing the LL diagram of our film with that calculated for high-quality bulk bismuth [28], a relation of $1.18\left[B_{i, \text { calc }}(\varphi)\right]^{-1}=\left[B_{i, \text { expt }}(\varphi)\right]^{-1}$ is established, in which $B_{i}$ is the magnetic field that the Fermi level lies at the center of the $i$ th LL. Since $\left[B_{i}(\varphi)\right]^{-1}$ is proportional to the cross section of the $i$ th electron pocket, the electron density in bismuth here is $n=1.18^{3 / 2} n_{\mathrm{b}}=3.85 \times 10^{17} \mathrm{~cm}^{-3}$, assuming the electron pockets are ellipsoidal. Here, $n_{\mathrm{b}}=3.0 \times 10^{17} \mathrm{~cm}^{-3}$ is the electron density of bulk bismuth [7]. Applying a similar method, the hole density is extracted to be $p=2.34 \times$ $10^{17} \mathrm{~cm}^{-3}$ from the LLs of the hole pocket [25].

With the $n$ and $p$ determined from the LL diagram at $2 \mathrm{~K}$, and the documented values of $n=p=2.45 \times 10^{18} \mathrm{~cm}^{-3}$ at $300 \mathrm{~K}$ [9], the remaining five variables can be obtained by simultaneously fitting two sets of AMR and PHE data along two current directions under the same $T$ and $B$ conditions. The semiclassical model described above can reasonably well fit the AMR and PHE curves up to $1 \mathrm{~T}$ at $2 \mathrm{~K}$ and up to $14 \mathrm{~T}$ at $300 \mathrm{~K}$, as shown in Fig. 2. The obtained mobility 


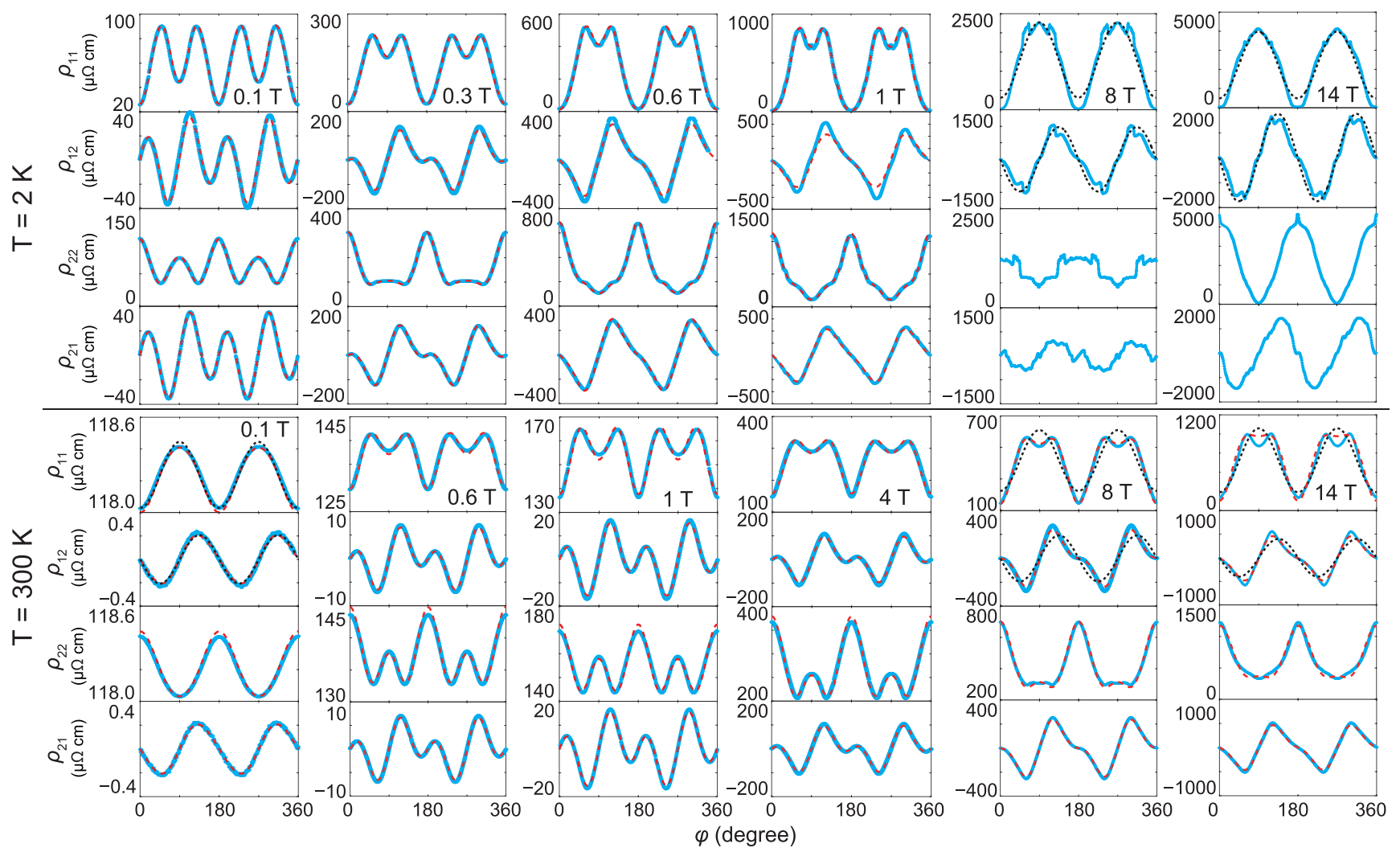

FIG. 2. Experimental AMR and PHE curves (solid blue) at 2 and $300 \mathrm{~K}$ in various magnetic fields from 0.1 to $14 \mathrm{~T}$, as indicated at the top of each column of figures. Fits to the experimental data with the semiclassical transport model are shown as dashed red curves and the chiral anomaly model as dotted black curves.

tensor elements as a function of $B$ are presented in Fig. 3, which reflect the highly anisotropic electronic structure of bismuth: $\mu_{1}$ is two orders of magnitude larger than $\mu_{2}$, and $\nu_{1}$ is one order of magnitude larger than $\nu_{3}$. These fits result in the largest mobility tensor element $\mu_{1}$ to be of the order of $10^{6} \mathrm{~cm}^{2} / \mathrm{V} \mathrm{s}$ at $2 \mathrm{~K}$. It should be noted that an even higher mobility and a larger PHE were observed in bulk bismuth [10], most likely because the carrier mean free paths are limited by the thickness in our bismuth films (anisotropic mean free paths ranging from 0.49 to $5.1 \mu \mathrm{m}$ along different directions) [25]. Furthermore, we notice that the mobility tensor elements in Fig. 3 vary with $B$, which is likely a result of three factors. First, since the carrier density in bismuth is already very tiny, the modification of the electronic band structure as well as
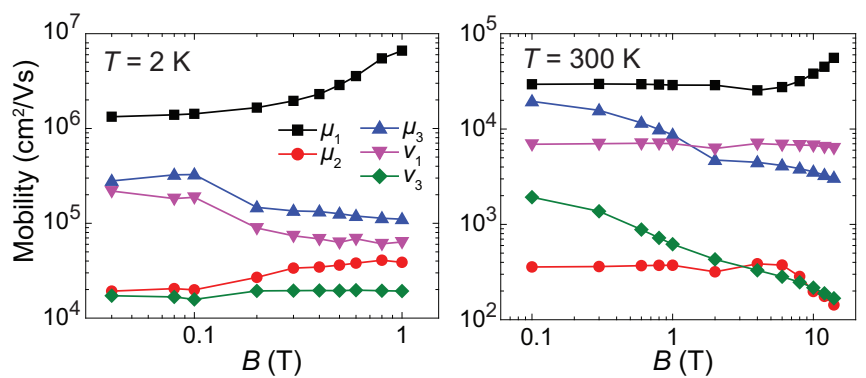

FIG. 3. Elements of the electron and hole mobility tensors at 2 and $300 \mathrm{~K}$, as obtained from fits with the semiclassical transport model. carrier densities by the formation of Landau levels cannot be neglected. Since the semiclassical model itself does not take Landau levels into account, this effect is absorbed within the magnetic field dependence of the mobility tensors. Second, as the mobility of bismuth is extremely high, the current jetting effect, which alters the current density distribution in the conduction channel, may come into play at high magnetic fields [10]. As the semiclassical model assumes the current density distribution to be uniform at any $B$, this effect is reflected in the variation of the mobility tensors. We will discuss the effect of current jetting further below. Finally, some anisotropic scattering processes might be sensitive to magnetic field [29]. More dedicated experiments are needed to confirm the specific scattering mechanisms.

The good agreement between the experimental data and the semiclassical model in low magnetic fields and at high temperatures suggests that these data can be explained by an anisotropic orbital origin. However, at $2 \mathrm{~K}$ and high field, the fitting results become less satisfactory. According to the semiclassical model, $\hat{\rho}$ tends to saturate at high fields when there is a difference between $n$ and $p$ [see Fig. S9 of the Supplemental Material (SM) [25]], while, by contrast, the experimental data exhibit no sign of saturation up to $14 \mathrm{~T}$. This can be understood from the LL diagram [Fig. 1(f)]: The quantum limit of electron pockets, at which all the electrons concentrate to the lowest LL, has been reached at $14 \mathrm{~T}$ at any $\varphi$ angle. The semiclassical model no longer holds when $B$ approaches the quantum limit. 
Since most PHE studies in nonmagnetic materials focus on novel Dirac or Weyl semimetals, in which a chiral anomaly is expected, while very few focus on semimetals that have trivial bulk states, it is useful to check whether our PHE data on bismuth thin films can be accounted for by a chiral anomaly model. Theoretical studies suggest that the chiral anomalyinduced AMR and PHE should have the form below [11,12],

$$
\rho(\varphi)=\left(\begin{array}{cc}
\rho_{1 \perp}-\Delta \rho_{1} \cos ^{2} \varphi & -\Delta \rho_{1} \sin \varphi \cos \varphi \\
\Delta \rho_{2} \sin \varphi \cos \varphi & \rho_{2 \perp}-\Delta \rho_{2} \sin ^{2} \varphi
\end{array}\right) .
$$

Obviously, the experimental data do not agree with Eq. (5) for every $T$ and $B$ condition, since Eq. (5) has a sinusoidal form and thus indicates two maxima on every AMR or PHE curve between 0 and $2 \pi$, while many curves in Fig. 2 exhibit four maxima. Nevertheless, under certain conditions (for instance, $T=2 \mathrm{~K}$ and $B=14 \mathrm{~T}$, or $T=300 \mathrm{~K}$ and $B=0.1 \mathrm{~T}$ ), $\rho_{11}, \rho_{12}$, and $\rho_{22}$ agree well with Eq. (5). However, the experimental $\rho_{21}$ has a phase shift of $\pi / 2$ comparing to Eq. (5), which would seem to clearly rule out a chiral anomaly mechanism. Under these conditions, if only $\rho_{11}$ and $\rho_{12}$ are measured in the binary channel, a positive conclusion about the existence of a chiral anomaly might be drawn, especially when a negative longitudinal magnetoresistance induced by current jetting is simultaneously seen [10]. Therefore, it is important to apply current along different crystalline axes that are perpendicular to each other when relating PHE with a possible chiral anomaly.

To understand where there is agreement and where there is a discrepancy between the chiral anomaly model and the experimental data, and how these differences evolve with magnetic field in the binary channel, we define dimensionless deviation ratios as follows,

$$
\begin{aligned}
D_{\mathrm{AMR}} & =\frac{\int_{0}^{2 \pi}\left|\rho_{11}(\varphi)-\rho_{11, \mathrm{CA}}(\varphi)\right| d \varphi}{\int_{0}^{2 \pi}\left|\rho_{11}(\varphi)-\bar{\rho}_{11}\right| d \varphi}, \\
D_{\mathrm{PHE}} & =\frac{\int_{0}^{2 \pi}\left|\rho_{12}(\varphi)-\rho_{12, \mathrm{CA}}(\varphi)\right| d \varphi}{\int_{0}^{2 \pi}\left|\rho_{12}(\varphi)\right| d \varphi},
\end{aligned}
$$

in which $\rho_{i j, \mathrm{CA}}(\varphi)$ are the resistivity tensor elements calculated from the fits to Eq. (5), and $\bar{\rho}_{11}$ is the average of $\rho_{11}(\varphi)$ over $[0,2 \pi]$. The results are summarized in Fig. 4. For low magnetic fields where the magnitude of AMR and PHE are beyond the resolution of our instruments, semiclassical simulations based on the fitted mobilities at $B=0.1 \mathrm{~T}$ were applied in Fig. 4 (see Supplemental Note 7 in SM [25] for the details of the simulations). At $300 \mathrm{~K}$ below $B=0.1 \mathrm{~T}$ and at $2 \mathrm{~K}$ below $B=0.001 \mathrm{~T}$, the $\rho_{11}$ and $\rho_{12}$ curves yielded by the semiclassical model agree well with Eq. (5). As $B$ increases, a discrepancy starts to appear, as shown in Fig. 4. At $2 \mathrm{~K}$, the discrepancy appears at a magnetic field two orders of magnitude lower than that at $300 \mathrm{~K}$, which is consistent with the difference in mobilities: The mobility tensor elements at $2 \mathrm{~K}$ are generally two orders of magnitude higher than those at 300 K. (In Figs. S7 and S8 of SM [25], we present a detailed analysis of the influence of each mobility tensor element on the shape of the AMR and PHE curves.) Despite such deviations with increasing $B$, at higher fields $(B>0.08 \mathrm{~T}$ at $2 \mathrm{~K}$ and $B>1 \mathrm{~T}$ at $300 \mathrm{~K}$ ), the two deviation ratios $D_{\text {AMR }}$ and $D_{\text {PHE }}$ start to decrease, implying the curves tend

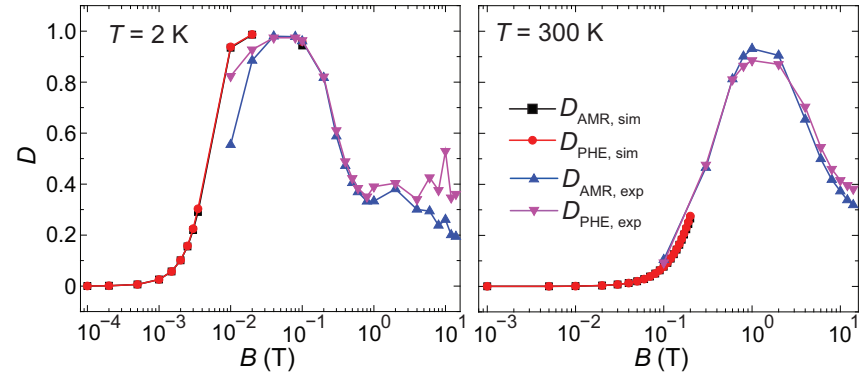

FIG. 4. Relative deviation between the chiral anomaly model and the experimental data or semiclassical model in the binary channel. The dimensionless deviation parameters $D_{\mathrm{AMR}}$ and $D_{\mathrm{PHE}}$ are defined in Eqs. (6) and (7). Part of the data at low fields was obtained from simulations based on the semiclassical model $\left(D_{\mathrm{AMR}, \mathrm{sim}}\right.$ and $\left.D_{\text {PHE,sim }}\right)$, in which the mobility tensors fitted from the experimental data at $B=0.1 \mathrm{~T}$ were adopted (see Supplemental Note 7 in SM [25] for details of simulations).

to assume shapes similar to those described by Eq. (5). The nonmonotonic magnetic field dependence of $D_{\mathrm{AMR}}$ and $D_{\mathrm{PHE}}$ can be understood as discussed below.

When $B$ is very low, the semiclassical model tends to yield similar sinusoidal shapes of the AMR and PHE curves as in Eq. (5), which is a coincidence between two different models. As $B$ increases, the anisotropic nature of the semiclassical model becomes more and more pronounced, thus the AMR and PHE curves deviate from the simple sinusoidal functions. At even higher $B$, the semiclassical model predicts a transition from four maxima/minima to two for the PHE curves (see Fig. S9 in SM [25]), which explains why two maxima/minima are seen again in the PHE curves above $B=0.6 \mathrm{~T}$ at $2 \mathrm{~K}$ and $B=8 \mathrm{~T}$ at $300 \mathrm{~K}$.

As for the data at $2 \mathrm{~K}$, the AMR curves show again a "sinusoidal-like" shape in analogy with Eq. (5) in high magnetic fields mainly because of the current jetting effect. As discussed above, current jetting, which makes the distribution of electric current density no longer uniform in the conduction channel, gradually becomes dominant as $B$ increases. When $\mathbf{E}$ and $\mathbf{B}$ are parallel (or antiparallel) to each other, the electric current tends to concentrate towards the center of the channel, leaving less current density at the edges, where the voltage electrodes pick up the signals [10]. Therefore, the AMR curves show two minima when $\mathbf{E}$ and $\mathbf{B}$ are parallel or antiparallel to each other, and two maxima when $\mathbf{E}$ and $\mathbf{B}$ are perpendicular to each other, resembling the sinusoidal function in Eq. (5) (for instance, the data at $2 \mathrm{~K}$ and $14 \mathrm{~T}$ in Fig. 2). Since the current jetting effect begins to appear at lower $B$ in systems with higher mobilities, $D_{\mathrm{AMR}}$ peaks at $B=0.08 \mathrm{~T}$ at $2 \mathrm{~K}$, a much lower field than $B=1 \mathrm{~T}$ at $300 \mathrm{~K}$.

Finally, we compare the PHE in our bismuth films with the other semimetals. In Fig. 5, the temperature dependence of the PHE in the bismuth thin films from this work is compared with those in other nonmagnetic semimetals, mostly bulk Dirac and Weyl semimetals, reported in the literature, typically at $B=14 \mathrm{~T}$. The bismuth film studied here has a large PHE of several $\mathrm{m} \Omega \mathrm{cm}$ over the whole temperature range of $2-300 \mathrm{~K}$, that is orders of magnitude larger than most of the PHE in materials reported to date. The topological semimetals with 


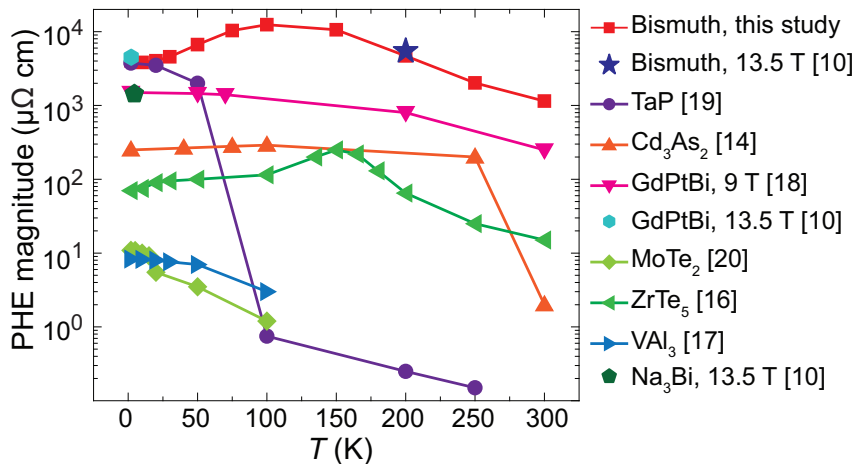

FIG. 5. Comparison between the temperature dependence of the magnitude of PHE in this study (the maximum of $2 \rho_{12}$ ) with other semimetals reported in the literature $\left[\Delta \rho_{1}\right.$ fitted from Eq. (5)]. The data were collected at $B=14 \mathrm{~T}$ unless specified in the figure.

the highest PHE at $2 \mathrm{~K}$, such as GdPtBi [10,18] and TaP [19], are of the same order of magnitude as our bismuth thin films. With increasing $T$, the magnitude of $2 \rho_{12}$ in our bismuth films [in comparison with $\Delta \rho_{1}$ in Eq. (5)] first increases, reaching a maximum of $12.4 \mathrm{~m} \Omega \mathrm{cm}$ at $14 \mathrm{~T}$ and $100 \mathrm{~K}$, and then decreases. This nonmonotonic behavior is likely due to the competition between a better compensation between $n$ and $p$ carrier densities and decreasing mobilities with increasing $T$.

In conclusion, we have demonstrated a very large PHE that reaches several $\mathrm{m} \Omega \mathrm{cm}$ at $14 \mathrm{~T}$ in several-micron-thick bismuth films grown by molecular beam epitaxy. The magnitude of the PHE in our bismuth thin films has a nonmonotonic temperature dependence, and the maximum value of PHE surpasses those reported in topological semimetals. Nevertheless, we conclude that the PHE can be largely attributed to an anisotropic orbital magnetoresistance based on detailed fits with a semiclassical transport model. Furthermore, a comparison between the experimental AMR and PHE data and a model based on the chiral anomaly in Dirac and Weyl semimetals shows agreement in the binary channel under certain $T$ and $B$ conditions, while in the bisectrix channel, a phase shift of $\pi / 2$ in the PHE is seen, which does not agree with the model. Thus we conclude that very large PHE can arise from orbital anisotropy in high-mobility semimetals.

We thank K. G. Rana, S. Zhou, M. Wang, H. Meyerheim, and I. Kostanovskiy for help with the sample characterizations, and M. N. Ali for useful discussions. This work was funded by Deutsche Forschungsgemeinschaft (DFG, German Research Foundation)-Project No. PA 1812/2-1.
[1] H. X. Tang, R. K. Kawakami, D. D. Awschalom, and M. L. Roukes, Phys. Rev. Lett. 90, 107201 (2003).

[2] A. Fernández-Pacheco, J. M. De Teresa, J. Orna, L. Morellon, P. A. Algarabel, J. A. Pardo, M. R. Ibarra, C. Magen, and E. Snoeck, Phys. Rev. B 78, 212402 (2008).

[3] A. Schuhl, F. Nguyen Van Dau, and J. R. Childress, Appl. Phys. Lett. 66, 2751 (1995).

[4] L. Ejsing, M. F. Hansen, A. K. Menon, H. A. Ferreira, D. L. Graham, and P. P. Freitas, Appl. Phys. Lett. 84, 4729 (2004).

[5] A. D. Henriksen, B. T. Dalslet, D. H. Skieller, K. H. Lee, F. Okkels, and M. F. Hansen, Appl. Phys. Lett. 97, 013507 (2010).

[6] P. N. Granell, G. Wang, G. S. Cañon Bermudez, T. Kosub, F. Golmar, L. Steren, J. Fassbender, and D. Makarov, npj Flexible Electron. 3, 3 (2019).

[7] Z. Zhu, B. Fauqué, K. Behnia, and Y. Fuseya, J. Phys.: Condens. Matter 30, 313001 (2018).

[8] R. Hartman, Phys. Rev. 181, 1070 (1969).

[9] J. P. Michenaud and J. P. Issi, J. Phys. C: Solid State Phys. 5, 3061 (1972).

[10] S. Liang, J. Lin, S. Kushwaha, J. Xing, N. Ni, R. J. Cava, and N. P. Ong, Phys. Rev. X 8, 031002 (2018).

[11] S. Nandy, G. Sharma, A. Taraphder, and S. Tewari, Phys. Rev. Lett. 119, 176804 (2017).

[12] A. A. Burkov, Phys. Rev. B 96, 041110(R) (2017).

[13] H. Li, H.-W. Wang, H. He, J. Wang, and S.-Q. Shen, Phys. Rev. B 97, 201110(R) (2018).

[14] M. Wu, G. Zheng, W. Chu, Y. Liu, W. Gao, H. Zhang, J. Lu, Y. Han, J. Zhou, W. Ning, and M. Tian, Phys. Rev. B 98, 161110(R) (2018).

[15] F. C. Chen, X. Luo, J. Yan, Y. Sun, H. Y. Lv, W. J. Lu, C. Y. Xi, P. Tong, Z. G. Sheng, X. B. Zhu, W. H. Song, and Y. P. Sun, Phys. Rev. B 98, 041114(R) (2018).

[16] P. Li, C. H. Zhang, J. W. Zhang, Y. Wen, and X. X. Zhang, Phys. Rev. B 98, 121108(R) (2018).
[17] R. Singha, S. Roy, A. Pariari, B. Satpati, and P. Mandal, Phys. Rev. B 98, 081103(R) (2018).

[18] N. Kumar, S. N. Guin, C. Felser, and C. Shekhar, Phys. Rev. B 98, 041103(R) (2018).

[19] J. Yang, W. L. Zhen, D. D. Liang, Y. J. Wang, X. Yan, S. R. Weng, J. R. Wang, W. Tong, L. Pi, W. K. Zhu, and C. J. Zhang, Phys. Rev. Materials 3, 014201 (2019).

[20] D. D. Liang, Y. J. Wang, W. L. Zhen, J. Yang, S. R. Weng, X. Yan, Y. Y. Han, W. Tong, W. K. Zhu, L. Pi, and C. J. Zhang, AIP Adv. 9, 055015 (2019).

[21] Q. Liu, F. Fei, B. Chen, X. Bo, B. Wei, S. Zhang, M. Zhang, F. Xie, M. Naveed, X. Wan, F. Song, and B. Wang, Phys. Rev. B 99, 155119 (2019).

[22] J. Meng, H. Xue, M. Liu, W. Jiang, Z. Zhang, J. Ling, L. He, R. Dou, C. Xiong, and J. Nie, J. Phys.: Condens. Matter 32, 015702 (2020).

[23] P. Li, C. Zhang, Y. Wen, L. Cheng, G. Nichols, D. G. Cory, G.-X. Miao, and X.-X. Zhang, Phys. Rev. B 100, 205128 (2019).

[24] D. L. Partin, J. Heremans, D. T. Morelli, C. M. Thrush, C. H. Olk, and T. A. Perry, Phys. Rev. B 38, 3818 (1988).

[25] See Supplemental Material at http://link.aps.org/supplemental/ 10.1103/PhysRevResearch.2.022029 for more information on film growth and device characterization, complete set of AMR and PHE fittings with semiclassical and chiral anomaly model, and details of the semiclassical model simulation.

[26] F. Y. Yang, K. Liu, K. Hong, D. H. Reich, P. C. Searson, and C. L. Chien, Science 284, 1335 (1999).

[27] Z. Zhu, A. Collaudin, B. Fauqué, W. Kang, and K. Behnia, Nat. Phys. 8, 89 (2012).

[28] Z. Zhu, B. Fauqué, Y. Fuseya, and K. Behnia, Phys. Rev. B 84, 115137 (2011)

[29] B. Fauqué, X. Yang, W. Tabis, M. Shen, Z. Zhu, C. Proust, Y. Fuseya, and K. Behnia, Phys. Rev. Materials 2, 114201 (2018). 